wenn mit kleinen Mengen gearbeitet wird; ein Einfluß des Gasmediums wurde nicht beobachtet.

4. 6 Gewichtsprozente Salzzusatz wurden als Minimum für die tiefste Färbung festgestellt.

5. Als Temperaturminimum der Darstellung wurde der Schmelzpunkt des zugesetzten. Salzes erkannt, während bei Erhöhung der Temperatur der Salzzusatz vermindert werden darf.

6. Es wurde der Einfluß des Wassergehalts bei niederen Temperaturen ähnlich demjenigen der Salze bei höheren Temperaturen befunden.

7. Der hierdurch wahrscheinlich gewordene Einfluß der Korngröße auf die Färbung wurde eindeutig bewiesen durch Uberführung der. braunen und violetten Farbe in das gelbrote Ausgangsprodukt mittels des umgekehrten Vorgangs der Feinkörnung infolge kombinierten Zerreibens und Sehlämmens.

\section{Über Störungen bei der Vereinigung von Stickoxyd und Sauerstoff.}

\author{
Von A. Mande und F. Russ. \\ (Vorläufige Mitteilung.) \\ Mit 1 Figur und 2 Tabellen.
}

(Eingeg. d. 13./11, 1907.)

Als wir das Verhalten der gasförmigen Untersalpetersäure (dem im Polymerisationsgleichgewicht befindlichen Gemenge von $\mathrm{NO}_{2}$ und $\overline{\mathrm{N}}_{2} \mathrm{O}_{4}$ ) gegen Wasser experimentell in Angriff nahmen, um das hierbei zu erwartende Gleichgewicht zwischen Salpetersäure, salpetriger Säure und Stickoxyd zu bestimmen ${ }^{1}$ ), gelangten wir bei anscheinend gleichen Versuchsbedingungen zu variablen Endzuständen.

Wir hatten hierbei die Untersalpetersäure durch Zusammenbringen stöchiometrischer Mengen von trockenem Stickoxyd und Sanerstoff bei Zimmertemperatur dargestellt, da wir nach den Angaben der, Literatur eine nahezu vollständige Vereinigung beider Gase erwarteten. Das Stickoxyd wurde nach dem bekannten, $L$ u $n$ g e schen, von $E \mathrm{~m}$ i c $\mathrm{h}$ 2) modifizierten Verfahren aus Nitrosylschwefelsäure und Quecksilber entwickelt, und der Sauerstoff zunächst durch Elektrolyse von Wasser gewonnen. Zur Durchführung der Reaktion bedienten wir uns pipettenförmiger GlasgefäBe (vgl. A in der Figur S. 487) von bekanntem Volumen, die zunächst mit Stickoxyd gefüllt wurden. Sodann wurde entsprechend der Reaktionsgleichung

$$
\begin{aligned}
& 2 \mathrm{NO}+\mathrm{O}_{2}=2 \mathrm{NO}_{2} \\
& 2 \mathrm{Vol} \text {. } 1 \text { Vol. } 2 \mathrm{Vol} .
\end{aligned}
$$

die Hälfte des Stickoxydvolumens an Sauerstoff zugefügt. Nach der Abkühlung auf Zimmertemperatur war in unserem Glasgefäße ein Minderdruck zu erwarten, da gemäß der Umsetzung

$$
2 \mathrm{NO}_{2} \rightleftarrows \mathrm{N}_{2} \mathrm{O}_{4}
$$

eine teilweise Polymerisation des primär gebildeten

1) Vgl. hierzu A. W. S s a pos ch nikow, Chem. Zentralbl. 1901, II, 1331; Cl. M o n te martini, Rendic. Acc. Lincei 6, 263 (1890); V. H. Veley, Chem. News 66, 175 (1892).

2) Wiener Monatshefte 13, 73 (1892).
Stickstoffoxydes zum Stickstofftetroxyd eintritt, dessen Größenordnung bei den herrschenden Versuchsbedingungen aus den $\mathrm{Nath}$ a $\mathrm{n}$ s on schen Zahlen ${ }^{3}$ ) über dieses Gleichgewicht zu ermitteln war.

Als wir sodann die Untersalpetersäure mit abgemessenen Wassermengen in Reaktion treten ließen, konnten wir trotz mehrfacher Wiederholungen bei anscheinend gleichen Bedingungen (wobei die zu analysierenden Proben zu gleichen Zeiten nach Versuchsbeginn entnommen wurden) zu übereinstimmenden Werten nicht gelangen, und dies auch dann nicht, als wir - um eventuelle Störungen durch Verschiedenheit der Gefäßwände zu vermeiden - die Versuche in ein und demselben Reaktionsgefäß durchführten. Das molekulare Verhältnis von Salpetersäure zu salpetriger Säure in der wässerigen Lösung, welches zur Beurteilung der Versuchsergebnisse zunächst herangezogen wurde, erwies sich hierbei als durchaus inkonstant.

Wir haben aus diesem Grunde von weiteren Bestimmungen abgesehen und bemühten uns vielmehr, die Ursache dieser Abweichungen aufzudecken. Die beobachtete Divergenz im Endzustande der Reaktion ließ sich schlieBlich auf Grund orientierender Versuche darauf zurückfübren, daß die primäre Vereinigung von Stickoxyd und Sauerstoff zu Untersalpetersäure nicht in allen Fällen, wie angenommen, fast voll ständig abläuft, sondern oft sehr viel früher zum Stillstand kommt.

Auffallenderweise zeigte sich, da $B$ dieser Endzustand nicht so sehr, wie vielleicht zu erwarten, von der Trocknung der Gase, als vielmehr von der Provenienz des zur Reaktion verwendeten Sauerstoffes abhängig zu sein scheint. Denn die Verwendung von aus Luft gewonnenem Sauerstoff (Bombensauerstoff von $\mathrm{L}$ in d e) führte zu einem fast vollkommenen Ablaufe der Reaktion, während z. B. ein aus Bariumsuperoxyd durch Umsatz mit $\mathrm{Ka}$ liumbichromat and Schwefelsäure erzeugter Sauerstoff die Reaktion viel früher zum Stillstand brachte. Bei Verwendung von durch Elektrolyse von Wasser gewonnenem Sauerstoff ergab sich eine fast vollständige Vereinigung, falls der Sauerstoff über erhitzten Palladiumasbest geleitet wurde; unterließ man dies, so war der Vereinigungsgrad geringer. Letztere Beobachtung ließ die Vermutung aufkommen, daß die Gegenwart von Ozon dem Ablaufe der Reaktion hindernd in den Weg tritt.

Durch willkürliche Anreicherung des Sauerstoffes an Ozon konnte der experimentelle Beweis erbracht werden, dab die Gegenwart von Ozon in der Tat einen nur unvollkommenen Ablauf der Reaktion :

$$
2 \mathrm{NO}+\mathrm{O}_{2}=2 \mathrm{NO}_{2}
$$

verursacht.

Wir lassen nunmehr jene orientierenden Versuche folgen, die wir in der erwähnten Richtung angestellt haben. Die verschiedenen Endzustände wurden auf manometrischem Wege ermittelt. Die hierbei gewählte Versuchsanordnung ergibt sich aus der Zeichnung und bestand aus dem pipetten-

3) Vgl. z. B. Ne r n s t, Theoretische Chemie 5. Aufl. S. 448; eine übersichtliche Tabelle über dieses Gleichgewicht findet sich bei v. J ü p $t \mathrm{n}$ e $r$, Lehrbuch der physikalischen Chemie I, 106. 
förmigen Reaktionsgefäß A von ca. $100 \mathrm{ccm}$ Inhalt, das mit einem einfachen Hahne $\mathrm{H}_{1}$ und einem Zweiweghahn $\mathrm{H}_{2}$ versehen war. Letzterer gestattete eine Verbindung des ReaktionsgefäBes mit Außen und eine solche mit dem Manometer (M), welches anfänglich durch einen mit Quecksilber abgedichteten Glasschliff in Verbindung mit A stand. Später wurde das Manometer an den Hahn $\mathrm{H}_{2}$ angeschmolzen. Das Manometerrohr selbst war mit Quecksilber beschickt, über dem wenig Nitrobenzol geschichtet war, um den Angriff des Quecksilbers durch das Peroxyd abzuschwächen. Demselben Zwecke diente die capillare Verengung des Rohres bis zur Marke K. Durch den Zweiweghahn $H_{2}$ konnte die Verbindung zwischen Manometer und dem Reaktionsgefä $ß$ jederzeit unterbrochen werden, was schon deshalb wünschenswert erschien, um den Aufbrauch des Peroxydes durch das Quecksilber möglichst zu verkleinern; doch konnte derselbe nicht ganz vermieden werden. Versuche, bei denen an Stelle des gewöhnlichen Manometers ein Fadenmanometer ${ }^{4}$ ) verwendet wurde, konnten nicht fortgesetzt werden, da ein Angriff des Quecksilbers nicht zu verhüten war, wodurch das Manometer gegen Druckdifferenzen unempfindlich wurde.

Für die Zweeke der Analyse, auf die wir später zu sprechen kommen, war an den Hahn $\mathbf{H}_{1}$ ein längeres Rohr $\mathrm{r}$ angesetzt.

Die Arbeitsweise gestaltete sich wie folgt :

Zunächst wurde mit Hilfe

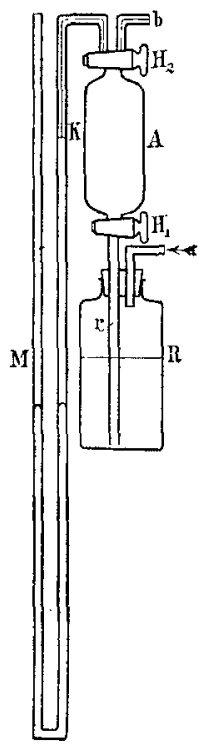
einer Wasserstrahlpumpe das

Gefäß A tunlichst evakuiert und sodann durch das Capillarrohr b die Verbindung mit dem Stickoxydentwickler hergestellt. Es wurde so lange frisch entwickeltes Stickoxyd durchgeleitet, bis eine hinter dem Gefäß A entnommene Gasprobe sich als 100\%ig erwies (Prüfung durch Absorption mit Eisensulfat). Sodann wurde der untere Hahn geschlossen, nach einigen Sekunden der obere, so daß im Reaktionsgefäße ein kleiner Utberdruck herrschte. Durch rasches Wenden des Hahnes $H_{1}$ wurde Atmosphärendruck innerhalb des Gefäßes hergestellt, Barometerstand und Temperatur abgelesen und durch den Hahn $\mathrm{H}_{2}$ der Sauerstoff, und zwar die Hälfte des Stickoxydvolumens, aus einer mit getrocknetem Quecksilber als Sperrflüssigkeit beschickten Gasbürette zugeführt. Zu dem Zweake wurde der Sauerstoff in der Bürette durch Heben des Niveaugefäßes unter Druck gestellt, so daB auch in jenen Fällen, wo die Vereinigung von Stickoxyd und Sauerstoff nur teilweise erfolgte, der gesamte Sauerstoff in kürzester Zeit eingebracht werden konnte. Es trat dann augenblicklich eine Rotfärbung in allen Teilen des Gefäßes ein, die je nach der Konzentration des entstandenen $\mathrm{NO}_{2}$ verschiedene Intensität aufwies. Doch waren bei ein

4) Vgl. Tam mann und Nernst, Z. physikal. Ch. 9, 6 .

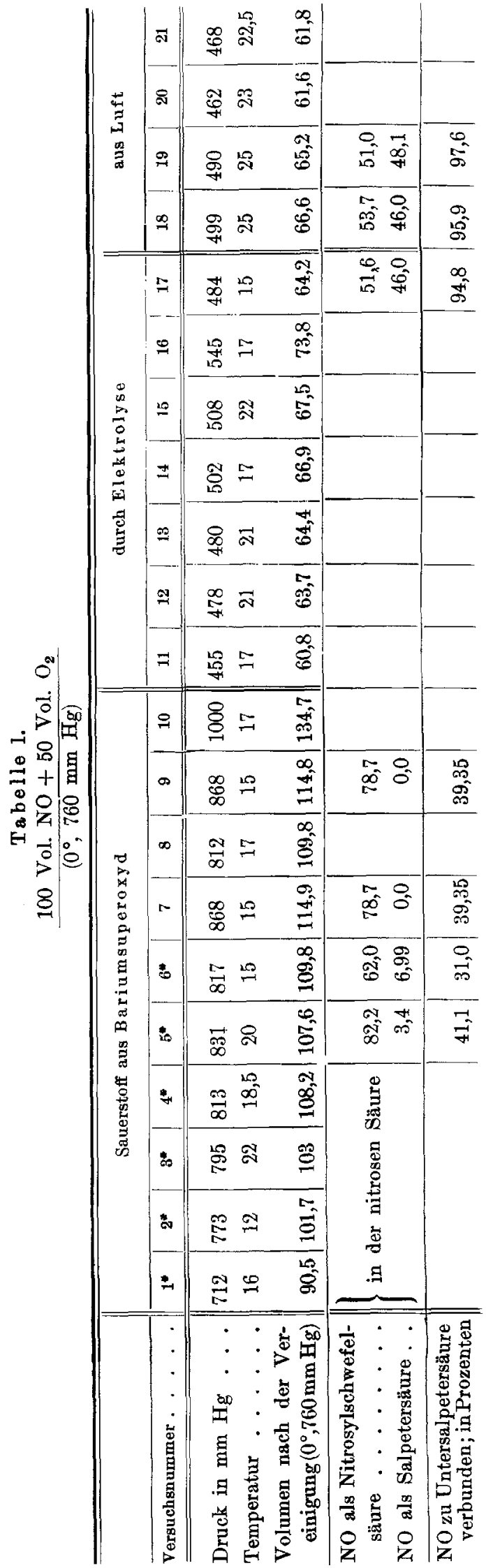


Tabelle 2.

Volumenprozentischer Gehalt des Gasgemisches $\left(\mathrm{N}_{2} \mathrm{O}_{4}+\mathrm{NO}_{2}\right)$ an $\mathrm{NO}_{2}$.

\begin{tabular}{c|c|c|c|c}
\hline \hline $\begin{array}{c}\text { Nummer } \\
\text { des } \\
\text { Versuches }\end{array}$ & toC & $\begin{array}{c}\text { Partial. } \\
\text { Druck in } \\
\text { Atmo- } \\
\text { sphären }\end{array}$ & \multicolumn{2}{|c}{$\%$ No } \\
beobachtet & berechnet \\
\hline \hline 19 & 25 & 0,60 & 36,93 & 38,9 \\
18 & 25 & $\mathbf{0 , 5 8}$ & 36,82 & 39,3 \\
17 & 15 & 0,55 & 26,86 & 28,5 \\
9 u. 7 & 15 & 0,24 & 35,5 & 37,6
\end{tabular}

und demselben Versuche innerhalb der Beobachtungszeiten Nuancierungen in der Farbe nicht wahrzunehmen, indem das Gasgemisch selbst nach Stunden dieselbe Färbung hatte, wio kurz nach dem Zusammentritt seiner Komponenten. Die Manometerablesungen erfolgten zu Anfang des Versuches und wurden periodisch so lange vorgenommen, bis nach Abkühlung des Systems der Druck konstant blieb 5 ).

In der Tabelle 1 (S. 487) befindet sich eine Zusammenstellung von Versuchen, bei denen Sauerstoff verschiedener Provenienz zur Verwendung gelangte. Vermerkt ist die Art der Trooknung, indem die Versuche, welche mit feuchten Gasen angestellt wurden, mit einem Stern (*) versehen sind, während bei allen übrigen Versuchen $\mathrm{P}_{2} \mathrm{O}_{5}$-getrocknete Gase verwendet wurden; ferner Druck und Temperatur im Reaktionsgefäße nach Stillstand der Reaktion und das aus diesen Größen unter Berücksichtigung sämtlicher Korrekturen berechneteVolumen, reduziert auf $0^{\circ}$ und $760 \mathrm{~mm} \mathrm{Hg}$. Die Zahlen beziehen sich auf 100 Volumenteile NO, die in Reaktion traten. Z. B zeigt Versuch 1 , daB $100 \mathrm{com}$ Stickoxyd und $50 \mathrm{ccm}$ Sauerstoff (auf $0^{\circ}$ und $760 \mathrm{~mm} \mathrm{Hg}$ reduziert) nach ihrer Vereinigung bei $16^{\circ} 90,5 \mathrm{ccm}$ (auf dieselben Daten reduziert) einnahmen.

Aus dieser Zusammenstellung ergibt sich die bereits einleitend erwähnte Beobachtung, daß die Reaktion zwischen Stickoxyd und Sauerstoff in fast allen. Fällen unvollständig abgelaufen ist. Setzen wir vollständigen Ablauf der Reaktion zu $\mathrm{NO}_{2}$ voraus und nehmen ferner an, daß sich letztere mit $\mathrm{N}_{2} \mathrm{O}_{4}$ im $\mathrm{N}$ ath a ns on schen Gleichgewichte befindet, so sollte das Gas bei einer Temperatur von $18^{\circ}$ ein Volumen von 58,5 ccm einnehmen ${ }^{6}$ ). Man

5) Um die Abkühlungszeit zu bestimmen, wurden besondere Versuche angestellt, bei denen sich im Reaktionsgefä $B$ ein Thermometer befand. $\mathrm{Es}$ ergab sich beim Zusammenbringen von NO und $\mathrm{O}_{2}$ eine maximale Temperaturerhöhung von $10^{\circ}$. Nach Ablauf von höchstens 10 Minuten herrschte innerhalb des Gefäßes wieder Außentemperatur. Eine Änderung am Manometer konnte nach dieser Zeit nicht mehr beobachtet werden.

6) Man gelangt zu dieser Zahl durch folgende Uberlegung auf bekannter Grundlage :

Ist von $1 \mathrm{Mol} \mathrm{N}_{2} \mathrm{O}_{4}$ der Bruchteil $x$ dissozüert, so befinden sich im Gasgleichgewicht entsprechend der Umsetzung

$$
\mathrm{N}_{2} \mathrm{O}_{4} \rightleftarrows 2 \mathrm{NO}_{2}
$$

neben (l-x) Molen $\mathrm{N}_{2} \mathrm{O}_{4}$ noch $2 \times$ Mole $\mathrm{NO}_{2}$, im ganzen somit $(1+x)$ Mole. ersieht, daß jene Versuche, bei welchen Luftsauerstoff verwendet wurde, sich noch am meisten diesem vollständigen Ablaufe nähern, daß sodann die mit elektrolytischem Sauerstoff vorgenommenen Versuche zwar beträchtliche Schwankungen untereinander zeigen, daB aber die Vereinigung bis ca. 94,8\% (Vers. 17) vorgeschritten ist. Am auffallendsten ist aber das Verhalten des aus Bariumsuperoxyd dargestellten Sauerstoffes, bei dem das Volumen nach dem Stillstande der Reaktion dasjenige des ursprünglichen überschreitet, und wo nur $39,35 \%$ (Vers. 7 u. 9) des NO sich in Untersalpetersäure verwandelten. Es war in diesen Fällen anzunehmen, daß neben Untersalpetersäure noch beträchtliche

Für das Gleichgewicht bei einer Temperatur $t$ gilt die Beziehung:

$$
\left.\frac{\left(\mathrm{p}_{\mathrm{NO}_{2}}\right)^{2}}{\left(\mathrm{p}_{\mathrm{N}_{2} \mathrm{O}_{4}}\right)}=\mathrm{k}_{\mathrm{t}} \ldots . \mathrm{1}\right)
$$

wenn mit $p$ die resp. Partialdrucke und mit $k_{t}$ die Gleichgewichtskonstante der Massenwirkung für die Temperatur $\mathrm{t}$ bezeichnen wird.

Die Partialdrucke können wir ausdrücken durch :

$$
\mathrm{p}_{\mathrm{N}_{2} \mathrm{O}_{4}}=\left(\frac{1-\mathrm{x}}{1+\mathrm{x}}\right) \mathrm{P} ; \text { und } \mathrm{P}_{\mathrm{NO}_{2}}=\left(\frac{2 \mathrm{x}}{1+\mathrm{x}}\right) \mathrm{P} \text {, }
$$

wenn $\mathrm{P}$ den Gesamtdruck des im Gleichgewicht befindlichen Systems aus $\mathrm{NO}_{2}$ und $\mathrm{N}_{2} \mathrm{O}_{4}$ bedeutet.

Die Gleichgewichtskonstante ergibt sich sodann aus 1 . zu

$$
\left.\mathrm{k}_{\mathrm{t}}=\frac{4 \mathrm{x}^{2}}{1-\mathrm{x}^{2}} \cdot \mathrm{P} . \ldots 2\right)
$$

Für die Ermittlung von $P$ ist zu berücksichtigen, daß wir $\mathrm{NO}_{2}$ von Atmosphärendruck bei $\mathrm{kon}$ s t a n t e m Vo l u men zum Teil in $\mathrm{N}_{2} \mathrm{O}_{4}$ verwandeln. Denselben Gleichgewichtszustand erreichen wir offenbar, wenn wir uns das $\mathrm{NO}_{2}$ bei konstantem Volumen volls tä ndig zu gasförmigem $\mathrm{N}_{2} \mathrm{O}_{4}$ umgewandelt denken und dieses $\mathrm{N}_{2} \mathrm{O}_{4}$ von nun $1 / 2$ Atmosphäre Druck dissoziieren lassen. Wir erhalten dann aus je einem Mol. $\mathrm{N}_{2} \mathrm{O}_{4}$ von 0,5 Atmosphärendruck $(1+x)$ Mole, deren Gesamtdruck $0,5(1+\mathbf{x})$ Atmosphären betragen wird.

Es ist demnach:

$$
\left.P=0,5+\frac{x}{2} \cdot \ldots 3\right)
$$

Dies .in 2. eingesetzt, ergibt:

$$
\left.2 \mathrm{x}^{3}+\left(\mathrm{K}_{\mathrm{t}}+2\right) \mathrm{x}^{2}-\mathrm{K}_{\mathrm{t}}=0 . \ldots 4\right)
$$

Für z. B. $18,02^{\circ}$ ist $K_{t}=0,07103$. Zur Bestimmung von $K_{t}$ wurden die von $v$. J $\ddot{u} p$ t n e ${ }^{*}$ ) aus den Daten von $\mathbf{E}$, und $L$. Nath a $\mathrm{N}$ s on ermittelten Werte herangezogen.

Setzen wir in 4 . für $\mathbf{K}_{\mathrm{t}}$ obigen Wert ein, so gelangen wir $\mathrm{zu}$

$$
x^{3}+1,0355 x^{2}-0,0355=0,
$$

woraus sich $x$ sehr annähernd zu 0,1702 berechnet.

Der Gesamtdruck P ergibt sich aus 3 . zu 0,5851 .

Unsere $100 \mathrm{ccm}$ NO von Atmosphärendruck hätten bei vollständigem Ablauf der Reaktion und konstantem Druck sich zu 58,51 ccm zu kondensieren. Dieses Gasgemisch setzt sich bei $0,5851 \mathrm{At}$ mosphärendruck zusammen aus $(1-x)$ Molen $\mathrm{N}_{2} \mathrm{O}_{4}$ und $2 x$ Molen $\mathrm{NO}_{2}$ oder in Volumenprozenten aus: $29,09 \% \quad \mathrm{NO}_{2}$ und $70,91 \% \quad \mathrm{~N}_{2} \mathrm{O}_{4}$. 2,98 .

*) v. Jü p t n e r, Lehrb. der phys. Chemie I, 
Mengen unverbundenen Stickoxydes vorhanden sind. Dieser Unterschied machte sich schon in der Farbendifferenz kenntlich, indem bei den Ver. suchen mit Superoxydsauerstoff die Farbentönung auffallend heller war, als bei den übrigen. Unterwirft man das mit Bariumsuperoxydsauerstoff erhaltenẹ Gasgemenge der Kondensation durch feste Kohlensäure, so bemerkt man das Auftreten einer blauen Flüssigkeit, während bei der Kondensation jener Gasmischungen, welche durch die Wechselwirkung mit Luftsauerstoff oder elektrolytischem Sauerstoff entstanden sind, sich farblose oder nur schwachgelb gefärbte Krystalle abschieden. Da nun bekanntlich reines Stickstoffperoxyd bei tiefen Temperaturen sich zu einer festen farblosen Masse kondensiert, andererseits Gasgemenge, welche neben dem Peroxyd noch unverbundenes Stickoxyd enthalten, sich hierbei zu einer blauen Flüssigkeit kondensieren, so kann dieses Verhalten zur qualitativen Entscheidung der Frage, ob NO neben $\mathrm{NO}_{2}$ vorliegt, herangezogen werden ${ }^{7}$ ). Wir haben in diesem Sinne einige Versuche wie folgt durchgeführt: Nachdem sich ein stationärer Endzustand gebildet hat, wurde das Reaktionsgefä $B$ in feste Kohlensäure + Äther gestellt und die Farbe des Kondensates beobachtet. Sodann wurde durch Auftauen das Kondensat vergast und der Druck am Manometer abgelesen. Derselbe stimmte mit dem Anfangsdrucke überein.

tberall dort, wo das Manometer eine sehr unvollkommene Vereinigung anzeigte $\left(\mathrm{BaO}_{2}\right)$, wurde bei der Kondensation eine blaue Flüssigkeit, sonst nur schwach gelb gefärbte oder farblose Krystalle beobachtet.

Der variable Endzustand, der bei Verwendung verschiedenen und auch ein und desselben Sauerstoffes gefunden wurde, ließ es nicht zu, diesen $\mathrm{Zu}$ stand als ein "Gleichgewicht" anzusprechen, vielmehr hatte es den Ansehein, daß eine Hemmung der Reaktion vorlag. Im Falle eines Gleichgewichtes hätte nach Entfernung der Untersalpetersäure die Reaktion zwischen Stickoxyd und Sauerstoff fortschreiten müssen. Zu diesem Zwecke wurde in mehreren Fällen konz. Schwefelsäure in das Reaktionsgefä $B$ so lange eingebracht, bis der nach der $A b$ sorption der Untersalpetersäure verbleibende Gasrest: unter Atmosphärendruck stand.

Das Einbringen der Schwefelsäure in das Reaktionsgefä $B$ erfolgte durch das Ansatzrohr $r$, das vorher ganz mit der Säure gefüllt wurde. Beim Offnen des Hahnes $H_{1}$ wurde, falls im Gefä $\beta$ Minderdruck herrschte, die Schwefelsäure selbsttätig eingesogen. Hersshte aber infolge sehr unvollkommener Vereinigung U’berdruck, so mußte zunächst etwas Säure eingepreßt werden ${ }^{8}$ ), die die Untorsalpetersäure absorbierte, wodurch ein Minderdruck entstand, und weitere Säure in das Gefäß treten konnte.

Eine weitere merkliche Vereinigung des über Schwefelsäure befindlichen Gasrestes konnte selbst nach einigen Stunden manometrisch nicht keobachtet werden; der Endzustand entsprach demnach nicht einem Gleichgewichte.

i) Vgl. hierzu die Messungen N. v. Wit t o r f s, Z. anorg. Chem, 41, 85 (1904).

8) Zum Einpressen diente die mit Schwefelsäure teilweise gefüllte Druckflasche $\mathbf{R}$.
Durch diescs Verhalten erschien es aber möglich, den Vereinigungsgrad des Stickoxydes zu Untersalpetersäure analytisch festzulegen, zunächst unter der Annahme, daß im Endzustande nur folgende Komponenten vorhanden sind: NO, $\mathrm{O}_{2}, \mathrm{NO}_{2}$ und $\mathrm{N}_{2} \mathrm{O}_{4}$. Konz. Schwefelsäure nimmt von diesen Gasen die gesamte Untersalpetersäure und vom Stickoxyd höchstens so viel auf, als dem molekularen Verhältnis $\mathrm{NO}_{2}$ : $\mathrm{NO}$ entspricht, da bekanntlich ein solches Gemenge sich gegen konz. Schwefelsäure wie $\mathrm{N}_{2} \mathrm{O}_{3}$ verhält.

Während reine Untersalpetersäure mit Schwefelsäure unter Bildung gleicher Moleküle Salpetersäure und Nitrosylschwefelsäure zusammentritt, führt das molekulare Gemenge von $\mathrm{NO}_{2}+\mathrm{NO} \mathrm{zu}$ Nitrosylschwefelsäure. Im Falle aber, daß mehr Mole $\mathrm{NO}_{2}$ als $\mathrm{NO}$ vorhanden sind, tritt eine geringfügige Komplikation hinzu. Es wäre zu erwarten, daß in diesem Falle die $\mathbf{g}$ e $\mathbf{s} \mathbf{a} \mathbf{m}$ t e $\mathbf{n}$ Stickoxyde von der Schwefelsäure aufgenommen werden, wobei das dem $\mathrm{N}_{2} \mathrm{O}_{3}$ entsprechende Gasgemenge von $\mathrm{NO}_{2}+\mathrm{NO}$ in Nitrosylschwefelsüure übergeht, während der verbleibende Rest an Untersalpetersäure zu gleichen Molen $\mathrm{HNO}_{3}$ und Nitrosylschwe. felsäure gelöst wird. Dem gegenüber zeigte sich aber in allen diesbezüglichen Fällen, daß nach der $\mathrm{Ab}$ sorption mit Schwefelsäure ein kleiner Gasrest verblieb, dessen Volumen höchstens $3 \%$ des angewandten Stickoxyds betrug, und den wir als ein hälftiges Gemisch von $\mathrm{NO}$ und $\mathrm{O}_{2}$ ansehen, da derselbe von einer Eisensulfatlösung mit der für Stickoxyd charakteristischen Braunfärbung gelöst wurde. Wir haben diesen Gasrest bei der Berechnung unserer Analysenerge bnisse mit berücksichtigt.

In allen Fällen, wie immer auch das molekulare Verhältnis von Untersalpetersäure zu Stickoxyd sein möge, ergibt eine Analyse der nitrosen Säure bei Bestimmung der Salpetersäure und der Nitrosylschwefelsäure die Menge des in Untersalpetersäure verwandelten Stickoxydes. Da nun das Volumen des angewandten Stickoxydes bekannt ist, so läßt sich aus beiden Größen der Vereinigungsgrad bestimmen.

FFeiterhin läßt sich aus den analytischen Daten der Dissoziationsgrad des $\mathrm{N}_{2} \mathrm{O}_{4}$ in $2 \mathrm{NO}_{2}$ ermitteln. Die in Betracht des orientierenden Zweckes nicht ganz exakton Messungen führen $\mathrm{zu}$ einer befriedigenden t'bereinstimmung, mit den aus den Werten von $\mathrm{E}$. und $\mathrm{L}$. $\mathrm{N}$ a $t$ a $\mathrm{n} \mathrm{s}$ o $\mathrm{n}$ durch $\mathrm{v}$. J ü $\mathbf{p}$ t n e $\mathbf{r}$ über dieses Gleichgewicht berechneten. Dies läßt den Sehluß zu, daß tatsächlich im Endzustande unserer Reaktionen in me $\beta \mathrm{barer}$ Konzentration nur die Gase $\mathrm{NO}, \mathrm{O}_{2}, \mathrm{NO}_{2}$ und $\mathrm{N}_{2} \mathrm{O}_{4}$ vorhanden waren.

Wir wollen die Art der Rechnung an Hand zweicr Versuche erläutern, und zwar einmal für den Fall einer nur teilweisen (Versuch $\mathrm{Nr} .7$ ) und zweitens für den Fall einer fast vollkommenen Vereinigung (Vers. Nr. 18).

1. Unvollkommener Ablauf, Ver such Nr. 7.: $100 \mathrm{ccm}$ Stickoxyd und 50 ccm Sauerstoff geben nach Ablauf der Reaktion ein Volumen von 114,9 (alle Daten auf $0^{\circ}, 760 \mathrm{~mm}$ reduziert). Es wurde Schwefelsäure eingepreßt, geschüttelt, und weiter Schwefelsäure so lange zugelassen, bis innerhalb des Gefäßes Atmosphärendruck herrschte. In der Schwefelsäure wurde nitrometrisch das Ge- 
samtstickoxyd bestimmt und mit Berücksichtigung der Löslichkeit von Stickoxyd in Schwefelsäure im Mittel $78,7 \mathrm{ccm} \mathrm{NO}\left(0^{\circ}, 760 \mathrm{~mm}\right)$ gefunden. Durch Einfließenlassen der nitrosen Säure in vorgelegtes $1 / 10^{-n}$. $\mathrm{KMnO}_{4}$ bis zur Entfärbung wurde der Gehalt an Nitrosylschwefelsäure bestimmt. Hierbei wurden ebenfalls $78,7 \mathrm{ccm}$ NO $\left(0^{\circ}, 760 \mathrm{~mm}\right)$ gefunden.

Das besagt, daß ein molekulares Gemenge von $\mathrm{NO}_{2}$ und $\mathrm{NO}$ mit der Schwefelsäure reagierte ${ }^{9}$ ). In unserem Falle sind dies $\frac{78,7}{2} \mathrm{ccm}$ NO, und derselbe Betrag an Stickoxyd liegt als Untersalpetersäure $\left\langle 2 \mathrm{NO}_{2} \longleftrightarrow \mathrm{N}_{2} \mathrm{O}_{4}\right\rangle$ vor. $\mathrm{Da}$ wir aber von $100 \mathrm{ccm}$ NO ausgingen, so müssen in der Gasphase auBerdem weitere $(100-78,7)=21,3$ ccm unverbundenes NO enthalten gewesen sein. An Sauerstoff waren ursprünglich $50 \mathrm{ccm}$ vorhanden, die zum Teil in $\mathrm{NO}_{2}$ resp. $\mathrm{N}_{2} \mathrm{O}_{4}$ übergingen. Dieser Anteil ergibt sich zu $\frac{78,7}{4} \mathrm{com} \mathrm{O}_{2}$. Der unverbundene Anteil $\mathrm{O}_{2}$ betrügt dann $\left(50-\frac{78,7}{4}\right) \mathrm{ccm}$.

Die Gaszusammensetzung nach Stillstand der Reaktion ergibt sich - ausgedrückt in Kubikzentimeter von $0^{\circ}$ und $760 \mathrm{~mm} \mathrm{Hg}$ - zu:

$39,35 \mathrm{ccm} \mathrm{NO}_{2}$ bzw. $19,67 \mathrm{ccm} \mathrm{N}_{2} \mathrm{O}_{4}$

(ohne Berïcksichtigung der Dissoziation.)

$$
\begin{array}{ll}
60,65 \mathrm{ccm} & \mathrm{NO} \\
30,33 \mathrm{~cm} & \mathrm{O}_{2}
\end{array}
$$

Für die Bestimmung des in $\mathrm{NO}_{2}$ dissoziierten $\mathrm{N}_{2} \mathrm{O}_{4}$ ziehen wir das manometrisch bestimmte Volumen heran. Dasselbe betrug $114,9 \mathrm{ccm}$. Wir fanden dasselbe aus den analytischen Daten ohne Berücksichtigung der Dissoziation zu 110,65. Die Differenz von $4,25 \mathrm{~cm}$ bedeutet die Volumenzunahme durch Dissoziation. Es dissoziieren demnach unter den obwaltenden Druck- und Temperaturverhältnissen $\quad 19,67 \mathrm{ccm} \quad \mathrm{N}_{2} \mathrm{O}_{4} \quad$ zu $\quad 23,92 \mathrm{ccm}$ $\left(\mathrm{N}_{2} \mathrm{O}_{4}+\mathrm{NO}_{2}\right)$.

Um den Gehalt an $\mathrm{N}_{2} \mathrm{O}_{4}$ und $\mathrm{NO}_{2}$ auszuwerten, überlegen wir, daß, falls von a ccm $\mathrm{N}_{2} \mathrm{O}_{4} \times$ dissoziieren, $2 \times \mathrm{NO}_{2}$ entstehen, so daß die Beziehung gilt : $(\mathrm{a}-\mathrm{x})+2 \mathrm{x}=\mathrm{b}$, woraus $\mathrm{x}=\mathrm{b}-\mathrm{a}$, wenn $b$ das gemessene End- und a das analytisch bestimmte Anfangsvolumen der Untersalpetersäure bedeuten :

In unserem Falle sind

$$
\begin{array}{rc}
\mathrm{x}=23,92-19,27 & =4,25 \\
\mathrm{~N}_{2} \mathrm{O}_{4}=\mathrm{a}-\mathrm{x}=\ldots & 15,42 \\
\mathrm{NO}_{2}=2 \mathrm{x}=\ldots & 8,5
\end{array}
$$

Mit Berükksichtigung der Dissoziation des $\mathrm{N}_{2} \mathrm{O}_{4}$ ergibt sich die prozentische Zusammensetzung des Gases im Endzustande zu :

$$
\begin{aligned}
& 52,8 \% \mathrm{NO} \\
& 26,4 \% \mathrm{O} \\
& 13,4 \% \mathrm{~N}_{2} \mathrm{O}_{4} \\
& 7,4 \% \mathrm{NO}_{2} \\
& \hline 100,0 .
\end{aligned}
$$

9) Vgl. L u n g e und B e r I, Z. angew. Chem. 19, 857 (1906) und $\mathrm{R}$ as ch ig, ebenda 21, 716 (1907).
$\mathrm{Um}$ zu sehen, inwieweit unsere Dissoziation des $\mathrm{N}_{2} \mathrm{O}_{4}$ mit den Zahlen von $\mathbf{N}$ a t a $\mathbf{n}$ s o $\mathbf{n}$ stimmt, berücksichtigen wir, daß der Partiuldruck des $\left(\mathrm{N}_{2} \mathrm{O}_{4}+\mathrm{NO}_{2}\right)$ in unserem Gasgemenge 20.8871 $=0,24 \mathrm{Atm}$. bei der Versuchstemperatur von $15^{\circ}$ beträgt. Die volumenprozentische Gaszusammensetzung der Untersalpetersäure ist $64,5^{\circ} / \mathrm{N}_{2} \mathrm{O}_{4} ; 35,5 \%$ $\mathrm{NO}_{2}$, während aus den Zahlen, welche v. J ü $\mathrm{p}$ $n$ e ${ }^{10}$ ) aus den Daten von $N$ a t a $n$ s o $n$ kritisch berechnet hat, sich die volumenprozentische $\mathrm{Zu}$ sammensetzung des Gasgemisches bei $15^{\circ}$ and 0,24 Atm. Druck zu : $62,4 \% \quad \mathrm{~N}_{2} \mathrm{O}_{4}+37,6 \% \mathrm{NO}_{2}$ ergibt.

2. Fast vollständiger Ablauf. Für den Fall eines fast vollständigen Ablaufes der Reaktion zwischen Stickoxyd und Sauerstoff tritt, wie schon erwähnt, eine kleine Komplikation ein. Man sollte erwarten, daß sich nunmehr das gesamte ursprünglich angewandte Stickstoffoxyd in der Absorptionssäure findet. Statt dessen zeigt der analytische Befund der Säure, wie auch die Prüfung des Gasrestes, daß kleine Quantitäten NO unverändert blieben. Stets findet sich in solchen Lösungen ein kleiner Uberschuß an salpetriger Säure. Dieser Ubberschuß könnte von einer Reduktion gebildeter Salpetersäure durch unverändertes Stickoxyd herrühren. Wir neigen aber mehr der Annahme zu, daß ein Teil der Untersalpetersäure unter Bildung von Nitrosylschwefelsüure reagierte.

Nun fanden wir bei Versuch 18:

Gesamtstickoxyd $99,72 \mathrm{ccm}$ von $0^{\circ}$ und $760 \mathrm{~mm} \mathrm{Hg}$. cem $\mathrm{NO}$ als $\mathrm{N}_{2} \mathrm{O}_{3} 53,72=46,02+7,70$

ccm $\mathrm{NO}$ als $\mathrm{N}_{2} \mathrm{O}_{4} 46,02$.

also $7,70 \mathrm{ccm}$ NO mchr Nitrosylschwefelsäure als Salpeterschwefelsäure, die entsprechend dem früher Gesagten aus $3,86 \mathrm{~cm}$ NO und $3,86 \mathrm{~cm} \mathrm{NO}_{2}$ resp. $1,93 \mathrm{ccm} \mathrm{N}_{2} \mathrm{O}_{4}$ entstanden sein muBten. Es verbleiben demnach $92,04 \mathrm{cem} \mathrm{NO}$ als $\mathrm{NO}_{2}$ resp. $\mathrm{N}_{2} \mathrm{O}_{4}$.

Demnach bestand das Gasgemenge aus $46,02+1,93=47,95 \mathrm{ccm} \mathrm{N}_{2} \mathrm{O}_{4}$ (ohne Berücksichtigung der Dissoz.)

$$
\begin{array}{lll}
4,10, & \text { NO } \\
2,05 & , & O
\end{array}
$$$$
1,7, \text {, N (von der Verunrei- }
$$
nigung des Sauer. stoffs stammend)

$55,80 \mathrm{~cm}$.

Das tatsächlich gemessene Gasvolumen betrug 66,62 und die Differenz : $66,62-55,80=10,82 \mathrm{ccm}$ bedeutet auch hier die Volumenzunahme durch Dissoziation.

Die Untersalpetersäure besteht somit aus:

$$
\begin{aligned}
& 37,13 \mathrm{ccm} \mathrm{N}_{2} \mathrm{O}_{4} \\
& 21,64,, \quad \mathrm{NO}_{2} .
\end{aligned}
$$

Die prozentische Zusammensetzung des Gases im Endzustand ist :

$$
\begin{array}{ccc}
55,7 \% & \mathrm{~N}_{2} \mathrm{O}_{4} \\
32,5 \% & \mathrm{NO}_{2} \\
6,2 \% & \mathrm{NO}^{2} \\
2,5 \% & \mathrm{O} \\
3,1 \% & \mathrm{~N} \\
\hline 100,0 &
\end{array}
$$

10) loc. eit. 
Der Partialdruck der Untersalpetersäure beträgt $=0,58 \mathrm{Atm}$., und ihre volumenprozentische Zusammensetzung betrug bei 0,58 at. und $t=25^{\circ}$

$$
36,8 \% \mathrm{NO}_{2}+63,2 \% \mathrm{~N}_{2} \mathrm{O}_{4} .
$$

Die Zahlen von $\mathrm{Natanson-Jüptner} \mathrm{er-}$ geben $39,3 \% \mathrm{NO}_{2}+60,7 \mathrm{~N}_{2} \mathrm{O}_{4}$.

Geht man von ungetrockneten Gasen aus (Vers. I-6), so tritt eine Komplikation ein durch Reaktion des Stickstoffperoxyds mit dem Wasserdampf der Gase, weshalb die vorstehenden Betrachtungen nicht ohne weiteres auf diese Versuche anwendbar sind.

In Tabelle 1 sind bei einigen Versuchen die analytischen Daten, gewonnen durch Analyse der nitrosen Säure, angeführt. Die letzte Horizontalreihe enthält den Vereinigungsgrad, ausgedrückt in Prozenten NO, die in Untersalpetersäure umgewandelt wurden.

Tabelie 2 enthält den beobachteten Gehalt der Untersalpetersäure an $\mathrm{NO}_{2}$ und den aus den Zahlen ron $\mathrm{Natanson} \mathrm{durch} \mathrm{v.} \mathrm{Jüptner} \mathrm{berechneten.}$ Die Ủbereinstimmung ist in erster Annäherung befriedigend.

Auf die Versuche, welche wir bisher beschrieben haben, wurden wir, wie eingangs erwähnt, rch dudas Verhalten des aus Stickoxyd und Sauer. stoff bestehenden Gemenges gegen Wasser geführt, wobei das in der Lösung ermittelte molekulare Verhältnis von $\mathrm{HNO}_{3}: \mathrm{HNO}_{2}$ unter anscheinend gleichen Bedingungen variierte. Wir fanden in allen Fällen dieses in Lösung sich einstellende Verhältnis zugunsten der salpetrigen Säure verschoben. In höchstem Maße war dies der Fall, wenn der Sauerstoff dem Bariumsuperoxyd entstammte. Dies erklärt sich aber nun zwanglos, da in der Gasphase mehr oder weniger unverbundenes Stickoxyd enthalten war, wodurch das Gleichgewicht der Reaktion ${ }^{11}$ ): $\mathrm{HNO}_{3}+2 \mathrm{NO}+\mathrm{H}_{2} \mathrm{O} \leftrightarrow 3 \mathrm{HNO}_{2}$ zugunsten der salpetrigen Säure verschoben wird.

Die bisher angeführten Versuche bringen die Tatsache zum Ausdruck, daß die Vereinigung von Stickoxyd je nach der Provenienz des Sauerstoffes zu verschiedenen Endzuständen führt. Die Deutung dieser Erscheinung begegnet Schwierigkeiten. Es erschien uns nicht unwahrscheinlich, daß geringe Mengen von Wasserstoffsuperoxyd, welche immerhin bei Gewinnung des aus Bariumsuperoxyd erzeugten Sauerstoffes in diesem enthalten sein könnten, eine Hemmung der Reaktion herbeiführen. Diese Annahme ließ sich experimentell nicht prüfen, da das Zufügen t r o e $\mathrm{k} \mathrm{e}$ n e n Wasserstoffsuperoxydes zum Sauerstoff nicht ausführbar war, und f e u ch te s Wasserstoffsuperoxyd nicht eingebracht werden kann, ohne eine rollständige Verschiebung des Endzustandes durch Absorption der Gase durch Wasser und Oxydation herbeizuführen. Leichter gelang es, zu prüfen, ob die Gegenwart von Ozon, welches sowohl in einem solchen Sauerstoff als im Elektrolytsauerstoff vorhanden sein könnte, die Reaktion hemmt. Wir gingen bei der Prüfung so vor, daß wir Parallelversuche ansetzten, wobei stets Stickoxyd derselben Partie und $\mathrm{P}_{2} \mathrm{O}_{5}$-getrockneter Lindesauerstoff verwendet wurden. In einem Falle wurde

11) Nach Vel e y, Chem. News 66 (1892). dieser Sauerstoff als solcher verwendet, im anderen Falle wurde er ozonisiert, bevor er mit dem Stickoxyd vereinigt wurde. Ließ man nun in beiden Fällen gleichzeitig in die beiden Reaktionsgefäße Wasser eintreten, bis Atmosphärendruck herrschte, so beobachtete man, daß das Reaktionsgefä $B$, in welchem nicht ozonisierter Sauerstoff zur Verwendung gelangte, bis auf ca. $10 \mathrm{ccm}$ sehr rasch gefüllt wurde, während in das zweite Gefäß, wo also ozonisierter Sauerstoff in Reaktion trat, das Wasser nur sehr langaam eindrang, bis schließlich nach $10 \mathrm{Mi}$ nuten ein stabiler Zustand sich einstellte; immerhin blieb dann ein Rest von ca. $40 \mathrm{ccm}$ unabsorbiert.

Wir erzeugten dann in beiden Gefüßen durch Abfließen des Wassers einen kleinen Minderdruck und saugten durch Öffnen der oberen Hähne Luft ein. Während im ersten Gefäße eine deutliche Rotfärbung eintrat, konnte dieselbe bei dem Gefäße, in welchem sich ozonisierter Sauerstoff befand, nicht beobachtet werden. Das Ergebnis dieser wiederholten Parallelversuche ist, daß Ozon der Vereinigung des Stickoxydes mit Sauerstoff hemmend entgegentritt.

Es erscheint uns nicht ausgeschlossen, daß die Vereinigung von Stickoxyd und Sauerstoff bei vollkommen reinen Gasen nur ungemein langsam vor sich geht, und daß die Gegenwart eines Katalysators für die rasche Vereinigung erforderllch ist. Wäre dies der Fall, so können unsere Beobachtungen auch so gedeutet werden, daß dieser Kataly. sator durch die Gegenwart von Ozon bzw. Wasserstoffsuperoxyd aufgebraucht wird, wodurch die Reaktion zum Stillstande käme. Dann dürfte die Reaktion - in Übereinstimmung mit der Beobachtung - auch dann nicht weiter fortschreiten, wenn die Untersalpetersäure der Gasphase entzogen wird ${ }^{12}$ ).

Obwohl die hier mitgeteilten Versuche keinesweges als abgeschlossen betrachtet werden können, glaubten wir, unsere Beobachtungen dennoch der Offentlichkeit übermitteln zu sollen, da äußere Verhältnisse eine Unterbrechung der Arbeit erfordern, und die mitgeteilten Beobachtungen vielleicht zur Aufklärung mancher Divergenz, welche sich über das Verhalten des Stickoxyds zum Sauerstoff $\mathbf{n}$ der Literatur findet, beitragen könnten.

Wien, Technologisches Gewerbe-Museum.

12) Da zwischen dem Verhalten des Luftsauerstoffes und dem ozonisierten ein wesentlicher Unterschied vorliegt, so bliebe $\mathrm{zu}$ erwägen, ob dem Luft. sauerstoff nicht ein spezifischer Katalysator eigen ist, der durch die Wirkung der stillen elektrischen Entladung verändert wird.

Bei der Einwirkung stiller elektrischer Entladungen auf Stickstoff-Sauerstoffgemische tritt neben Ozon noch ein gasförmiger Körper auf, den P. Hau tefeuille und J. Chappuis (Compt. rend. 32, 80 [1881]) für $\mathrm{N}_{2} \mathrm{O}_{6}$ ansehen. E. War burg und G. Leithäuser (Drudes Ann. 23, 209 [1907]) sehen diese Formel des gasförmigen Körpers nicht für begründet an. Sie finden unter anderem, daß dieser Körper auch durch Einwirkung von trockenem Ozon irgendwelcher Herkunft auf $\mathrm{N}_{2} \mathrm{O}_{5}$ erhalten wird und nennen ihn $\mathrm{Y}$. Da $\mathrm{N}_{2} \mathrm{O}_{5}$ durch Einwirkung von Ozon auf $\mathrm{N}_{2} \mathrm{O}_{4}$ entstehen kann, so folgt, daß auch in unserem Gasgemisch $\mathbf{Y}$ enthalten sein kann, das den Katalysator vielleicht zerstör. 